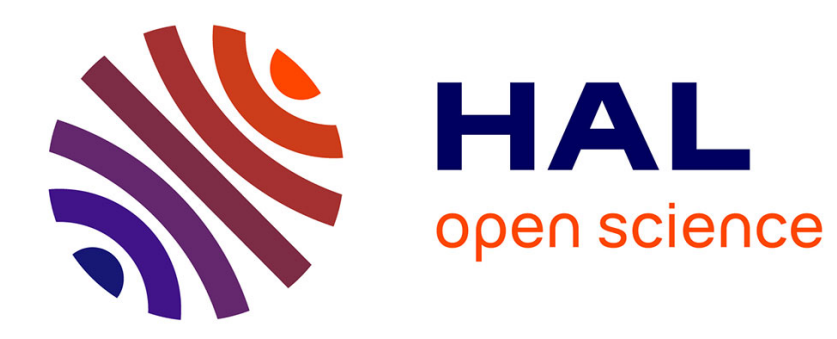

\title{
Eh bien comme évaluateur de discours
}

Florence Lefeuvre

\section{To cite this version:}

Florence Lefeuvre. Eh bien comme évaluateur de discours. Travaux de Linguistique: Revue Internationale de Linguistique Française, 2012, pp.123-143. halshs-01142361v2

\section{HAL Id: halshs-01142361 \\ https://shs.hal.science/halshs-01142361v2}

Submitted on 18 Apr 2015

HAL is a multi-disciplinary open access archive for the deposit and dissemination of scientific research documents, whether they are published or not. The documents may come from teaching and research institutions in France or abroad, or from public or private research centers.
L'archive ouverte pluridisciplinaire HAL, est destinée au dépôt et à la diffusion de documents scientifiques de niveau recherche, publiés ou non, émanant des établissements d'enseignement et de recherche français ou étrangers, des laboratoires publics ou privés. 
A citer sous cette forme : Lefeuvre F. 2012: «Eh bien » comme évaluateur de discours à l'oral (spontané ou représenté), Travaux de linguistique, Bien en perspective (Moline ed.), $\mathrm{n}^{\circ}$ 65 , p. 123-143.

\section{Eh bien comme évaluateur de discours}

L'objet de cet article est d'examiner bien comme évaluateur du discours, à l'oral spontané ou représenté, sous la réalisation du segment eh bien :

[1] spk3:[...] on avait des loyers assez bon marché on avait vraiment ici des gens euh de tous les milieux si tu veux qui payaient bien leur loyer donc des gens pas du tout euh + pas du tout dans la misère mais qui étaient vraiment des gens de de classe moyenne eh bien ils ont été obligés d' partir en banlieue (CFPP2000, 11-03 ${ }^{1}$ )

et plus précisément la façon dont il initie des unités syntaxiques prédicatives (ils ont été obligés d' partir en banlieue en [1]). Eh bien fait partie des «ponctuants » (Vincent 1993, Morel et Danon-Boileau 1998), «particule[s] énonciative[s]» (Fernandez-Vest, 1994), «marqueur[s] métadiscursif[s]» (Hansen 1995), «petite[s] marque[s] du discours » (Brémond 2002), «particule[s] discursive[s]» (Teston-Bonnard 2006), qui parsèment le discours oral. Nous parlerons pour notre part de «marqueurs discursifs », à l'instar de Dostie et Pusch 2007. Dans la typologie des marqueurs discursifs que nous répartirons en quatre grands groupes - les évaluateurs de discours (bon), les formes interactionnelles (écoute, tu vois, hein), les connecteurs logiques ou temporels (donc, alors), les lexèmes liés à la (re)formulation (disons, enfin, quoi) —, nous classerons eh bien dans les évaluateurs de discours. Eh bien est généralement répertorié comme marqueur discursif dans l'oral spontané. Il survient également dans l'oral représenté : dialogues de théâtre, dialogues romanesques, tout récit où le narrateur adopte une focalisation interne (Genette 1972) et plus généralement tout discours marqué explicitement par la présence du locuteur. C'est pourquoi, nous mènerons notre étude en nous appuyant sur deux types de corpus : i) des corpus d'oral spontané, celui du français parlé parisien (CFPP2000 : cf. Branca, Fleury, Lefeuvre, Pires 2009, avec 36 h d'enregistrement et 500000 mots (27 occurrences de eh bien) et celui de Clapi (http://clapi.univ-lyon2.fr, avec $45 \mathrm{~h}$ d'enregistrement (28 occurrences) ; ii) le corpus Frantext dont nous avons sélectionné 319 occurrences par ordre chronologique (de 1998 à 2011), parfois écrit en hé bien pour 19 occurrences. Nous nous proposons de montrer comment le sémantisme d'eh bien lié à l'évaluation du discours fait de lui un marqueur qui initie des unités syntaxiques prédicatives et comment se réalise son positionnement topologique en présence d'autres «préfixes » devant le "noyau » (i.e. sujet-verbe, cf. Le Goffic 2011 d'après Blanche-Benveniste 1997), avec des différences notables, en fonction du type de discours examiné (oral spontané vs oral représenté). Nous nous situons donc dans une perspective syntaxique du discours, selon le cadre théorique développé dans Le Goffic 2011.

\section{1. (Eh) bien comme évaluateur de discours}

Après avoir mis en exergue dans quelle mesure bien permet de valider des énoncés, qu'il se trouve inséré dans une phrase verbale ou averbale, nous verrons que le marqueur discursif $e h$ bien garde ce rôle d'évaluation du discours.

\footnotetext{
${ }^{1}$ Le premier chiffre donne le lieu, ici, le $11^{\mathrm{e}}$ arrondissement de Paris et le deuxième le classement de cet entretien parmi ceux du $11^{\mathrm{e}}$ arrondissement : il s'agit du troisième enregistrement effectué dans le $11^{\mathrm{e}}$ arrondissement de Paris.
} 


\subsection{Bien validant le discours, en phrase verbale ou averbale}

Les études dévolues à l'adverbe bien concernent principalement son emploi en phrase verbale. Les auteurs mentionnent régulièrement le rôle de bien dans la validation d'une assertion, ce qui nous semble essentiel pour notre propos ici. Ainsi Culioli 1988 évoque le cas de figure où bien opère sur une relation prédicative et permet de la valider, comme dans :

[2] Il est bien (effectivement) dans le tiroir (ex. tiré de Culioli 1988, p. 158)

et à sa suite, Péroz 1992 étudie les valeurs de validation de bien qui portent sur la convenance entre l'assertion et l'énonciation de celle-ci. Pour un exemple tel que [2], il parle d'un bien « confirmatif ». Parmi les valeurs de bien que Maj-Britt Mosegaard Hansen (1998a) relève, se trouve celle de la particule d'assertion, dans des emplois tels que :

[3] C'est bien la première fois que ça m'arrive (ex. tiré de Mosegaard Hansen 1998)

Selon Martin 1990, bien adverbe de phrase « signifie le caractère incontestablement valide de ce qui est dit » dans des cas de figure où il faut que soit (ré)affirmé ce caractère valide (par exemple, après une hésitation ou une mise en cause). Martin 1990 met également en avant le fait que bien est un inverseur de polarité et qu'il se combine difficilement avec des mots « qui se situent d'emblée dans le positif ». Dans le même ordre d'idée, Jacqueline Van BaardewijkRességuier (2002) évoque la tension que cet adverbe connaît, partagé qu'il est entre son aptitude à orienter l'énoncé vers le positif et à être implicitement porteur de négativité (cf. également Hansen : 1998a). Il initie un "parcours » entre ces deux pôles opposés. Nous verrons que cette valeur semble persister dans les emplois d'eh bien.

Lorsque bien revêt le rôle de prédicat averbal (cf. Lefeuvre 1999) au sein d'un énoncé, il permet de valider la relation prédicative prononcée dans un énoncé précédent, ce qui peut rappeler ses emplois dans la phrase verbale. Généralement, l'emploi de bien en tant que prédicat s'opère au sein d'un échange conversationnel :

[4a] spk2:j'pense que j'axerais mes mes recherches étant donné que quand on achète j'pense que c'est pour le long terme j'axerais mes recherches sur des quartiers euh:: seizième septième premier euh: huitième:

spk1 : d'accord +

spk2 : voilà +

spk1 : bon + très bien (CFPP2000, 07-02)

[5] spk2 : donc euh + c'est vrai que j'ai jamais c'est vrai que j'me souviens + de quasiment aucun nom de mes profs de de de collège mais alors euh:: lui il est resté

spk1: mmh mmh + + très bien + et euh: donc on parlait de:: les limites du du quartier précédemment + est-ce que vous voyez une différence::: entre le Paris-Est et le ParisOuest ++ (CFPP2000, 07-02)

Dans cet emploi, l'adverbe bien renvoie alors à une prédication $(\mathrm{P})$, qui correspond en [4a] et [5] à un tour de parole précédent. Nous dirons qu'il fonctionne en emploi résomptif (cf. Maillard 1974, Lefeuvre 2007). Dans ce type d'exemple, très bien permet de valider cette prédication :

"P (j'axerais mes recherches sur des quartiers euh:: seizième septième premier euh: huitième) est très bien",

"P (c'est vrai j'ai jamais c'est vrai que j'me souviens + de quasiment aucun nom de mes profs de de de collège mais alors euh:: lui il est resté) est très bien".

Il peut se trouver comme ici employé en proximité de bon voire de d'accord'. Ces termes peuvent également valider le discours précédent. La valeur prédicative de bien est encore perceptible par la présence de l'intensif très qui pourrait être supprimé. Winther 1985 note,

\footnotetext{
2 Pour plus détails sur le sens de ce bien, cf. Bouchaddakh S. \& Dostie G. 2007.
} 
dans des exemples tels que ci-dessus, la proximité de l'emploi de bien avec bon et met en lumière son fonctionnement de ponctuant discursif ou métadiscursif dans un discours particulier, le débat dirigé. Il est difficile effectivement, tout comme dans notre corpus, d'ajouter la négation pas :

[4b] : [...] j'axerais mes recherches sur des quartiers euh:: seizième septième premier euh: huitième:

? spk1 : pas très bien.

Il n'est possible d'introduire la négation pas que dans certains contextes, notamment lorsque bien porte sur le contenu, alors que précédemment il portait sur l'énonciation :

[6] spk1 : je lui ai tiré la langue

spk2 : pas bien!

On voit ainsi que bien a perdu en partie, dans des exemples tels que [4a] et [5], son aptitude à fonctionner comme un terme averbal prédicatif susceptible d'accueillir la négation (cf. Lefeuvre 1999). Cela correspond au fait que, sur le plan du sens, très bien constitue un simple accord formel sur un fait (cf. Winther 1985), qui permet à l'interviewer de proposer une autre question, comme on le voit dans l'exemple [5].

\subsection{Eh bien évaluant le discours}

Cette possibilité d'évaluer et de valider le discours n'a pas disparu, selon nous, dans les emplois d'eh bien. Il est possible d'associer l'emploi d'eh bien à celui de bien prédicatif, lorsqu'il constitue une unité isolée, délimitée par la ponctuation dans l'oral représenté :

[7a] spk2 : ils s-en fait ils sont arrivés y en a un qui m'a collé une baffe + comme ça + puis il commençait à s'éloigner (mm) moi je comprenais pas pourquoi c' qui se passe + donc le mec j'ai chopé bon je l'ai + j'lui ai réglé son compte $(\mathrm{mm})$ voilà et seul à seul ça allait + puis bon ses neuf petits autres camarades me sont tombés dessus d'un coup donc voilà

spk1 : eh bien + ils étaient du du même âge que vous à peu près?

spk2 : euh oui + ils devaient avoir un an de moins $j^{\prime}$ pense un an de moins un an d'plus en plus c'était dans ces eaux-là oui (CFPP2000, 20-02)

[8a] Pour finir, il me demande ce que je fais dans la vie. Quand je le lui dis, il fait une drôle de tête.

- Avocat? Eh bien! Et l'asthme ne vous gêne pas quand vous plaidez?

- Je ne fais jamais de crise d'asthme quand je plaide. (Winckler, La maladie de Sachs)

Le fait qu'eh bien forme une unité isolée révèle encore une certaine autonomie. Ce marqueur évalue le discours sur sa gauche, marquant une surprise - indignée ou au contraire admirative - , et peut se paraphraser par exemple par ça alors ou bien par des adjectifs prédicatifs $^{3}$ du type inoui, incroyable:

[7b] spk2 : ça alors / inoui / incroyable + ils étaient du du même âge que vous à peu près?

[8b] - Avocat? Ça alors / Inoui / Incroyable ! Et l'asthme ne vous gêne pas quand vous plaidez?

En revanche, dans des emplois tels que :

[9a] spk2: en même temps ils faisaient des fêtes extraordinaires sur le palier c' qu'on ferait plus maintenant un très très grand palier et lui eh bien la Russe blanche est partie il a passé ses examens il est entré très jeune à vingt-quatre ans au cabinet de Georges Mandel mais il est resté dans cet immeuble (CFPP2000, 07-03)

\footnotetext{
${ }^{3}$ Cf. Lefeuvre 1999, 2007 : nous considérerons que ces adjectifs, associés à une modalité d'énonciation, forment des unités syntaxiques autonomes.
} 
eh bien ne constitue plus une unité isolée mais introduit une unité coordonnée à une autre unité précédente, fonctionnant comme un «ligateur discursif » (Cf. Morel et Danon-Boileau 1998) ou un «marqueur discursif» (Dostie et Pusch 2007). Il a perdu également son rôle prédicatif. Certes il peut être remplacé par un adjectif prédicatif tel que incroyable:

[9b] et lui incroyable la Russe blanche est partie

mais à la différence du cas précédent, incroyable caractérise alors le discours de droite, pouvant se déplacer sans changer le sens :

[9c] et lui la Russe blanche est partie incroyable

Ce n'est pas le cas d'eh bien:

[9d] *et lui la Russe blanche est partie eh bien

Le sémantisme de bien mis en évidence plus haut et lié à la validation de ce qui précède semble avoir disparu, comme le montre cet exemple où c'est bien en tant qu'unité isolée qui valide ce qui précède et qui constitue un prédicat adverbial :

[10] je le trouve laid. Bon, dis-je. Vous n'avez pas autre chose? me demande-t-il.

$J^{\prime}$ avais aussi pensé, lui dis-je, à appeler ça La vie malaise. Eh bien très bien, La vie

malaise, dit-il, très bien, ça s'appellera comme ça. Mais cette fois, c'est moi qui ne veux pas. (Echenoz, Jérôme Lindon)

On peut toutefois se demander si eh bien a perdu la possibilité d'évaluer le discours. Nous ferons l'hypothèse d'une part qu'eh bien continue à évaluer le discours et d'autre part que c'est toujours le discours de gauche qu'il évalue. En effet, son inaptitude à se déplacer en fin d'énoncé (cf. [9d]) montre que sa portée concerne l'énoncé précédent et non le suivant. Le sens de bien ne valide plus ce qui précède mais prend en considération telle situation ou tel discours :

« considérons que ce qui est / a été dit»

en l'orientant vers ce qui suit, selon un schéma progressif du type :

" considérons telle chose et proposons quelque chose d'autre »

ce qui permet de dépasser ce qui a été dit précédemment. Il orienterait l'énoncé précédent vers le négatif (cf. 1.1. la valeur de bien) et introduirait un énoncé plus adéquat. Par ce mouvement, bien dans eh bien joue un rôle également par rapport au discours de droite. Cette analyse se rapproche de la valeur suivante d'eh bien distinguée dans Hansen (1998b) : selon cette auteure, une des principales valeurs d'eh bien est de signifier que le discours sur sa droite se comprend en fonction du discours qu'il initie sur sa gauche ; plus précisément, soit il indique ce qui est au moins temporairement accepté dans le discours de gauche, soit il indique que la pertinence de ce qui précède le marqueur est remise en question.

En ce qui concerne $e h$, ce mot garde sa valeur de marqueur d'interpellation comme dans cet exemple :

[11] Mais les corps se serraient à notre passage et bientôt nous nous retrouvâmes dehors, dans la neige fondue. Malgré le cri d'une vendeuse : " $\boldsymbol{E h}$, derrière la porte, n'attendez plus, il n'y en aura pas pour tout le monde! ", les gens continuaient à affluer. (Makine, Le testament français).

Il signale une interpellation sur un nouvel objet de discours, ce qui permet de focaliser l'attention sur ce qui suit. Nous avons donc, avec eh bien, une tension entre d'une part une évaluation de ce qui précède et d'autre part une focalisation sur ce qui suit. La combinaison de ces deux morphèmes, l'un portant sur la gauche et l'autre sur la droite, leur permet de délimiter des unités syntaxiques, comme nous allons le voir dans notre deuxième partie : c'est ce qui réunit les différents emplois syntaxiques d'eh bien comme marqueur discursif.

\section{Délimitation d'unités syntaxiques par eh bien}


Le fait que bien évalue le discours de gauche et que $e h$ focalise sur le discours de droite permet de comprendre qu'eh bien peut se trouver comme élément charnière de deux unités :

[12] " J'ai tué mon premier policier à l'âge de seize ans, dit cet homme à Karayan. Il me demandait mes papiers d'identité. Eh bien je l'ai abattu, c'est comme ça que je me suis identifié. " (Le coup de feu sur Véron, le chargeur vidé sur les épicières. (Perrut, Patria o muerte)

Cependant c'est son rôle de marqueur de frontière qui est régulièrement exploité dans le discours, et notamment la possibilité qu'il a d'initier des unités.

Voyons quels types d'unités il introduit.

\subsection{Les unités syntaxiques introduites par eh bien}

Eh bien délimite des unités syntaxiques prédicatives autonomes qui sont généralement verbales (exemple [12]). Il peut également initier des segments verbaux non autonomes comme une complétive, en discours oral représenté :

[13a] [...]Il bégaie donc un peu plus distinctement qu'euh eh bien ça ça lui ferait plaisir de de la revoir. (Benoziglio, La voix des mauvais jours et des chagrins rentrés) mais également en discours oral spontané :

[14]

est ce que (inaud.) pose la question est ce qu'il est pas humain d'accepter le fait que les personnes à qui on a demandé de l'aide eh bien après on leur rende non seulement on l eur rende en leur donnant cette nationalité mais en leur donnant l'occasion de pouvoir $r$ amener leurs proches pour (inaud.) (Corpus : Débat sur l'immigration, http://clapi.univ-lyon2.fr)

On peut alors se demander si ces segments deviennent autonomes. Il semble que non, comme le montre l'impossibilité d'insérer une modalité interrogative :

[13b] *Il bégaie donc un peu plus distinctement qu'euh eh bien est-ce que ça ça lui ferait plaisir de de la revoir?

Cela dit, en [13a], la présence du marqueur d'hésitation euh, de la répétition de ça et de de, ainsi que d'eh bien lui-même rapproche ce segment du discours rapporté direct, syntaxiquement plus autonome. Eh bien, en initiant des noyaux syntaxiques, rappelle le fonctionnement syntaxique d'autres marqueurs discursifs tels que bon qui est également un terme averbal évaluateur de discours.

Les unités syntaxiques autonomes initiées par eh bien peuvent être averbales (cf. Lefeuvre 1999) :

[15] je tombe sur une feuille de notes prises le 20 mars 1980 pour préparer... mon premier cours sur le journal! Vingt ans après! Eh bien, mes amis, quelle dégelée ! Je le traite de tous les noms... (Lejeune Philippe, Signes de vie, le pacte autobiographique 2)

On relève alors régulièrement la structure eh bien oui :

[16a] "Mais alors, vous êtes égoïste! », me dit-on comme si c'était quelque chose d'impardonnable. Eh bien oui, je suis égoïste. (Groult, Mon évasion, 2008)

[17] vous me prêtez un faible pour Altan Toloum, eh bien oui, c'est un homme que je tiens en grande estime. (Lanzmann, La Horde d'or)

Cet emploi s'explique par la perte de la valeur prédicative de bien, assurée en fait par oui qui permet de valider clairement le discours précédent. Oui peut alors se paraphraser par c'est vrai. Suit alors une reformulation de l'énoncé précédent, qui en modifie parfois à peine la teneur $[16 \mathrm{a}]$ : 
[16b] vous êtes égoïste / je suis égoïste.

Ou bien peut apparaître une justification de la phrase précédente, vous me prêtez un faible pour Altan Toloum en [17].

L'unité prédicative en oui peut se clôturer par quoi (cf. Lefeuvre et al. 2011), ici avec un infinitif disloqué en périphérie :

[18] Eda allait mourir. Eh bien oui, quoi, mourir. (Duras, Cahiers de la guerre et autres textes)

Il est possible de trouver eh bien non qui invalide le discours précédent, marquant une nette rupture par rapport à ce qui précède :

[19] spk1: j'avais un autre enfant j'avais rencontré quelqu'un hein ça s' passait + bien ou très bien au départ + et + ensuite j'ai bénéficié si on peut dire ça de mon chômage pour continuer à l'élever moi dans ma petite tête j'ai j'ai dit "bien + il aura un peu plus d'un an j'vais retrouver facilement du travail " eh bien non ça a été la grosse galère hein (CFPP2000, IV-01)

[20] Étoile entre les étoiles, il peut gravir tous les degrés de la gloire académique. Eh

bien non. Refus d'entrer à la Comédie-Française. Cinéma, télévision. Et Cyrano (Podalydès, Voix off)

Cela n'est pas vraiment contradictoire avec la présence d'eh bien puisque celui-ci ne valide pas le discours précédent mais prend juste en considération ce qui a été dit. En [20], l'énoncé d'après présente une assertion qui reformule ce qui précède en y intégrant une négation lexicale.

Eh bien peut se trouver avec d'autres prédicats averbaux marquant une approbation du discours précédent (eh bien tant mieux, eh bien d'accord) :

[21]Nage, promène-toi, fais la grasse matinée, tu reviendras transfigurée. Il ne veut pas

? Eh bien tant mieux! Tu t'imagines vivre avec lui, non mais, c'est une anomalie.

(Mréjen, Eau sauvage)

ou au contraire une mise à distance du discours antérieur :

[22] Allons, Rousseau est fou, tout cela est ridicule, c'est comme si moi je vous disais...

- Eh bien, tant pis, je vais vous le dire!

Je suis aussi fou que Rousseau : je crois qu'il dit vrai. (Lejeune, Signes de vie, le pacte autobiographique 2)

Le fait d'initier des unités syntaxiques prédicatives explique pourquoi eh bien se trouve de façon récurrente dans certaines configurations discursives.

\subsection{Des configurations discursives récurrentes}

Nous en avons relevé quatre.

i) Eh bien peut marquer la frontière gauche d'une nouvelle formulation, qui en suit une non aboutie. A l'oral spontané, il apparaît ainsi dans des cas de reprise :

[23] spk2 : [...] on allait en classe là et + à Notre-Dame de Sion donc ah oui j'avais j'étais petite parce que $j^{\prime}$ suis pas restée longtemps j'étais eh bien on on mettait nos cartables dans un triporteur de la poste et ils nous les parce que comme ça on pouvait être plus libres et ils nous les redonnaient à l'arrêt euh boulevard Raspail (CFPP2000, 07-03)

Il permet de proposer une nouvelle unité syntaxique prédicative. Dans l'oral représenté, nous avons trouvé un exemple où le locuteur cherche un mot; eh bien permet d'introduire la formulation trouvée par le locuteur : 
[24] [I1] avait balbutié qu'il y avait certainement, avec quelques aménagements, certes, certainement de quoi faire de la pièce quelque chose de très, oui, de très..., eh bien, de très gai et vivant. Tu connais la blague : trois chambres de bonnes et une de mauvaise, et elle avait dit Ah ah et, à nouveau, s'était pendue à son cou (Benoziglio, La voix des mauvais jours et des chagrins rentrés)

ii) Eh bien initie des tours de parole (oral spontané) :

[25] spk1: y a pas de solidarité entre Français / spk2 : euh:: du tout / spk3 : du tout

/spk2 spk3 : du tout c'est dommage

spk2 : non du tout que + eux ils en ont + ils en ont beaucoup +

spk1 : eh bien + espérons qu'à force de comités d'quartier:: [rires] et autres conseil de:: + quartier (CFPP2000, 12-03)

des répliques (oral représenté) :

[26] "Il y a de bonnes choses dans la pièce, me dit l'oncle Paul avec un clin d'oeil appuyé à ma mère, mais toi, tu joues comme un pied, ma pauvre Rosie! - Eh bien elle va continuer à donner des leçons particulières aux élèves retardés $d u \quad 7 e$ arrondissement, dit Maman. (Groult, Mon évasion)

Dans le corpus Frantext, on peut même trouver eh bien en tout début de dialogue:

[27] C'est alors que se passe cette vilaine chose : le sang rouge qui se met à monter en bouillonnant un peu dans la seringue, qui se remplit, se remplit.- Eh bien, vous en avez un beau sang! dit avec entrain la religieuse. L'enfant ne voit plus rien. (Sizun, Éclats d'enfance)

iii) Les réponses aux questions sont souvent introduites par eh bien. C'est le cas de l'oral spontané et de l'oral représenté. Mais l'oral spontané privilégie les réponses structurées sur un verbe (cf. Lefeuvre à paraître a)) :

[28] spk1: alors qui vient?

spk2 : euh eh bien on a toujours un peu ceux-là qui vieillissent euh y en a d'autres qui sont partis du quartier [...] (CFPP2000, 14-01)

[29] EF et alors cet cette espèce de dépression là qui était finalement très liée à la mort non puisque vous $x$ aviez envie de vous/vous en êtes_sortie comment alors?

FA17 eh bien je m'en suis sortie euh disons $\bar{b}$ on ben/ avec un traitement/ de cheval(Corpus : Français des années 80 - entretiens sociolinguistiques, http://clapi.univ-lyon2.fr)

alors que dans l'oral représenté les réponses sont généralement averbales (une seule réponse verbale sur 17 exemples; cf. également Lefeuvre et Tanguy à paraître) :

[30] C'est ça! J'ai l'impression qu'un éclair me traverse le bras et évidemment ça m'inquiète, à mon âge...

- À bon? À quoi penses-tu?

- Eh bien, à l'infarctus, pardi ! (Winckler, La Maladie de Sachs)

Un locuteur peut produire lui-même la question et la réponse et introduire celle-ci avec $e h$ bien, ce qui démarque bien les deux mouvements du couple question-réponse :

[31] spk3: qu'est-ce qu'on faisait quand on était une jeune fille de spk2: ah autrefois? Duruy?

spk3 : autrefois oui

spk2: qu'est ce qu'on faisait quand on était une jeune fille de Duruy? eh bien on allait on faisait partie des jeunesses musicales de France (CFPP2000, 07-05)

[32] d'un bon sourire et d'un haussement d'épaules fataliste : c'est un peu ridicule, bien sûr, mais c'est comme ça. Et l'autorisation, qui peut nous la donner? Eh bien, le FSB. 
Florence Lefeuvre - Université de la Sorbonne Nouvelle (Paris 3) - Clesthia Syled EA 2290

Je demande alors si le responsable du FSB est toujours un certain Sacha (Carrère, Un roman russe)

iv) Eh bien marque clairement la borne gauche du discours rapporté :

[33] " "ah oui Monsieur" "ça m'intéresse, je vous en offre tant, si toutefois vous acceptez la proposition...". Il nous donnait plus que le prix voulu! J'ai dit "eh bien Monsieur, il y a déjà quelqu'un qui est intéressé mais je vais réfléchir, je vous rappelle". Et c'est comme ça qu'on a vendu. (Mrejen Valérie, Eau sauvage)

L'exemple suivant met en évidence le fait qu'eh bien est associé au discours rapporté :

[34] [...] elle lui demande de répéter. Il bégaie donc un peu plus distinctement qu'euh eh bien ça ça lui ferait plaisir de de la revoir. Avec un petit sourire, les yeux brillants, elle dit qu'elle n'y verrait pas d'objection majeure. (Benoziglio, La voix des mauvais jours et des chagrins rentrés)

Il est perçu comme propre à l'oral spontané, de même que l'interjection euh et la répétition de ça et de.

Le fait qu'eh bien marque ainsi la frontière de nouvelles unités syntaxiques a des incidences sur le déroulement du discours.

\subsection{Incidences dans le déroulement du discours}

Plusieurs auteurs montrent qu'eh bien permet d'introduire quelque chose d'inattendu (cf. Sirdar-Iskandar 1980), comme dans l'exemple [19] avec eh bien non. A l'oral spontané, Young-ai Hwang (1993) présente l'hypothèse selon laquelle eh bien peut marquer une " rupture de consensualité », comme dans cet exemple où l'interviewer change de sa propre initiative de sujet :

[35] spk2 : c'est suffisamment vous n'êtes pas hystérique de la propreté

spk1: non + non

spk2 : et donc [...] vous trouvez que que ça suffit

spk1: oui j' trouve que ça va oui

spk2 : d'accord + eh bien euh écoutez j'crois que j' ai fait le tour de mes questions mais peut-être pas des vôtres vous vous attendiez peut-être à d'autres euh d'autres thèmes (CFPP2000, 11-01)

La rupture impliquée par eh bien peut être signifiée par d'autres changements linguistiques. Peuvent survenir par exemple des changements dans les temps employés ainsi que dans la forme du discours. Dans cet exemple, eh bien signale le passage d'une valeur aspectuelle sécante (imparfait), propre à délivrer un cadre, à un aoriste du discours (passé composé) approprié à la narration :

[36] " J'ai tué mon premier policier à l'âge de seize ans, dit cet homme à Karayan. Il me demandait mes papiers d'identité. Eh bien je l'ai abattu, c'est comme ça que je me suis identifié. » (Perrut, Patria o muerte)

La réplique suivante marque une rupture temporelle avec le passage du présent de l'indicatif, propre à constater une activité, au futur de l'indicatif avec ici une valeur de prophétie :

[37] Des Allemands s'adressant cet hiver à des femmes qui faisaient la queue, disaient méchamment :

- Ah! Vous vous plaignez! Eh bien, un jour viendra où vous mangerez de l'herbe. (Auroy, Ma vie sous l'Occupation)

Cette rupture peut se retrouver dans les actes de discours initiés par eh bien qui vont de la suggestion à l'ordre :

[38] elle nous surprend dans les travées, au fond, à ne rien faire, ou le faisant mollement, en compagnie des autres intérimaires, l'apostrophe est immédiate : " $\boldsymbol{E} \boldsymbol{h}$ 
bien, gros ballots, qu'est-ce que vous faites donc plantés là? Allez zou ! " Le " zou », sans appel, nous disperse comme une volée de moineaux (Podalydes, Voix off)

[39] Ils nous plaisent s'ils sont invisibles. S'ils ne font pas d'histoire. S'ils sont sans histoire.

Quant aux autres, ceux qui sont en trop, eh bien, qu'ils cessent d'entrer par la fenêtre alors qu'on les a chassés par la porte. (Storti, L'arrivée de mon père en France)

$\mathrm{Ou}$ bien notre marqueur peut s'associer à des adverbes ou pronoms négatifs, tels que personne, jamais et plus en [40]:

[40] ah !... L'ai entendu en Touraine, à la Grange de Meslay, dans les années, quoi ?, 75, jouer la Deutsch 960, ti-la-li-la-la, vous savez? Eh bien, personne, vous m'entendez ?, per-son-ne jamais plus n'a... (Benoziglio, La voix des mauvais jours et des chagrins rentrés)

Le forclusif plus implique également une « rupture », celle de la « continuité temporelle » (cf. Riegel et al.2009).

Nous avons vu jusqu'ici des exemples où eh bien introduit des unités syntaxiques prédicatives. Il peut se trouver en présence d'autres groupes de mots qui se trouvent comme lui devant le « noyau » (sujet-prédicat, Le Goffic 2011).

\section{Approche topologique d'eh bien comme préfixe}

En adoptant un point de vue topologique, nous considérerons eh bien comme un préfixe (cf. Le Goffic 2006 et 2011, d'après Blanche-Benveniste 1997), vu qu'il survient devant le noyau et jamais après. Ce marqueur peut apparaître avec d'autres préfixes. Deux possibilités se dessinent alors. Soit il se trouve en première position de l'ensemble des préfixes, soit il n'est pas en première position.

\subsection{Eh bien en position de premier préfixe}

Le positionnement d'eh bien comme premier constituant est présenté comme attendu, dans Morel et Danon-Boileau 1998 (p. 39), pour l'oral spontané : eh bien y est décrit comme un « ligateur énonciatif». Cela peut se comprendre également d'après la valeur que nous avons dégagée en 1 : eh bien évalue le discours sur sa gauche tout en ouvrant sur une nouvelle unité. L'oral spontané se caractérise par un ordre canonique des constituants du "préambule » (Morel et Danon-Boileau 1998) ou des préfixes (Blanche-Benveniste 1997), ce qui donne selon la première terminologie : ligateur, point de vue, modus dissocié, cadre, support lexical disjoint - et selon la deuxième terminologie : point de vue, lieu, temps, disloqué avec reprise (annexant un locatif ou temporel), disloqué sans reprise. Nous suivrons cette dernière typologie, qui se base davantage sur la syntaxe. Les marqueurs discursifs évaluateurs de discours, tels que bon et eh bien, se trouvent préférentiellement en tout début d'énoncé (cf. Lefeuvre 2011 pour bon), devant les éléments qui marquent le point de vue. Ils font partie des « ligateurs énonciatifs » de Morel et Danon-Boileau 1998.

Quelles formes peuvent se trouver après eh bien ?

Eh bien peut être suivi par des marqueurs discursifs qui appartiennent à des formes interactionnelles basées sur un verbe conjugué tel que écoutez et tu vois (cf. Leth Andersen H., 2007) :

[41] spk1: oui j' trouve que ça va oui

spk2 : d'accord + eh bien euh écoutez j'crois que j' ai fait le tour de mes questions mais peut-être pas des vôtres vous vous attendiez peut-être à d'autres euh d'autres thèmes (CFPP2000, 11-01) 
[42] Tu disais que je passerais mon certificat d'études et puis... et puis... que je serais une grande dessinatrice de mode. Eh bien, tu vois, ce n'est pas du tout comme ça. Je suis quelqu'un d'autre. (Szczupak-Thomas, Un diamant brut Vézelay-Paris 1938-1950)

Sans doute est-ce le cas parce que, dans ces exemples, bien porte sur le discours qui se trouve à sa gauche alors que $t u$ vois et écoutez portent sur le discours qui se trouve à leur droite. En revanche, lorsqu'eh bien survient dans un énoncé en même temps que le marqueur discursif bon évaluateur de discours, l'ordre semble être bon eh bien:

[43a] Il y a plusieurs centaines de boîtes de biscuits, ils les ont fait rouiller sur place, un peu trop. Bon, eh bien, elle est un peu trop rouillée! Ça n'a pas d'importance... (Boltanski, Grenier, La vie possible de Christian Boltanski) [43b] *h bien bon elle est un peu rouillée.

On peut faire l'hypothèse que cet ordre entre bon et eh bien est dû à la présence de $e h$ qui oriente eh bien sur la droite.

Sinon, peuvent survenir après eh bien :

- le marqueur de point de vue moi :

[44] On y prend goût, à la longue. Eh bien, moi, j'aime les pluies écossaises. Bref, j'aime l'Écosse. (Roubaud Jacques, Poésie : récit)

- un circonstant :

[45] spk2: non j'pense pas + non moi je + si je revois autour de moi + non parce que j'vois j'ai une soeur qui est née en quarante-quatre + eh bien en soixante-huit elle avait donc euh vingt-quatre ans + eh bien ça faisait un moment qu'elle + vivait qu'elle avait des + copains que ma mère trouvait ça tout à fait normal (CFPP2000, 07-05)

[46] Je pensais souvent à Henri, combattant quelque part en Afrique, en Italie, en France peut-être. Oh ! s'il savait ! Et s'il venait en permission! Eh bien, le mois dernier, il s'est trouvé brutalement en face de l'horrible vérité. (Auroy, Jours de guerre : Ma vie sous l'Occupation)

- un élément disloqué (ou support lexical disjoint ou encore élément thématique, ici avec reprise) :

[47] spk4 : les sorties à vélo c'était en Forêt d'Fontainebleau

spk1 spk3 : c'était plus ouais

spk4 : euh ils avaient que quinze quin- quinze places disponibles

spk1: XX quartier

spk4 : y a quinze eh bien les quinze personnes c'étaient que quinze personnes que du même quartier + et on était deux et j'avais été avec un XX c'était Youssef (CFPP2000, Saint-Ouen-02)

[48] on se surprend dans la glace d'un commerçant - féroce, la lumière du matin blême en hiver! -, et c'est le choc! "C'est moi, ça ? Pas possible! Il y a une erreur!" Eh bien cette erreur, on m'offrait de la réparer (enfin, le mot est inexact : ce n'est pas " offert » du tout), disons qu'on me proposait de m'enlever quinze ans (Groult, Mon évasion)

Lorsqu' eh bien est suivi de deux préfixes, nous avons trouvé comme possibilité :

- un marqueur de point de vue et un circonstant :

[49] - Élisabeth Badinter dans un article du Monde s'est dite "profondément humiliée par l'idée des quotas ».

- Eh bien moi, si je suis profondément humiliée, c'est par le fait que nous soyons la dernière démocratie d'Europe pour la représentation des femmes (Groulet, Mon évasion)

- un élément signalant un point de vue et un élément disloqué : 
[50] Tiens, j'ai vu quelque part sur la ligne de Sceaux, qui ne s'appelle plus ligne de Sceaux d'ailleurs, une sorte de hangar rempli d'anciens portillons du métro, eh bien, pour moi, tout cela, c'est une sorte de mémoire active. J'essaie de me souvenir, je me force à me souvenir. (Pérec, Entretiens et conférences II [1979-1981]) Dans l'oral représenté, nous trouvons donc les cas de figure répertoriés pour l'oral spontané dans Morel et Danon-Boileau 1998 et Blanche-Benveniste 1997.

C'est lorsqu'eh bien se trouve dans une autre position préfixale que des changements apparaissent entre l'oral spontané et l'oral représenté.

\subsection{Eh bien dans une autre position}

Lorsqu' eh bien n'apparaît pas en première position dans le préfixe, deux cas de figure peuvent être distingués. Soit eh bien peut survenir après un élément disloqué :

[51] «- Oui. On nous oubliera. C'est la vie, rien à faire. Ce qui aujourd'hui nous paraît important, grave, lourd de conséquences, eh bien, il viendra un moment où cela sera oublié, où cela n'aura plus d'importance. Et, c'est curieux, nous ne pouvons savoir aujourd'hui ce qui sera un jour considéré [...] »(Ernaux, Les Années)

Soit eh bien peut apparaître après un circonstant :

[52] Quand le 17 juillet, les bandes armées font une descente sur Sarzane, bastion rouge au milieu d'un territoire peu à peu conquis par les chemises noires, eh bien, les carabiniers font face et arrêtent onze des attaquants, dont leur chef Renato Ricci. (Storti, L'arrivée de mon père en France)

Dans ce cas, eh bien porte toujours sur le discours de gauche mais cette fois, non sur l'unité prédicative autonome précédente, mais sur l'élément disloqué ou les circonstants spatiotemporels. Généralement ces derniers développent alors des formulations complexes centrées sur un prédicat: la présence d'eh bien souligne le fait qu'elles se trouvent prises en considération par rapport à ce qui suit. Eh bien sert alors de frontière entre d'une part ce qui est pris en considération et d'autre part la nouvelle unité prédicative qu'il initie. Voyons plus précisément ces deux cas de figure où l'oral représenté et l'oral spontané ne présentent pas toujours le même type d'occurrences.

Le premier cas concerne l'élément disloqué qui correspond généralement au thème de l'énoncé. Dans l'oral représenté, nous trouvons régulièrement ce cas de figure, avec des thèmes complexes, comme en [51] avec la structure ce qui $P$ ou bien avec des éléments nettement détachés du reste de la phrase, que ce soit par la ponctuation :

[53] L'un était Mazarin, l'autre était Louis XIV : je préfère Louis XIV! La suite... Eh

bien ça renvoie à son histoire intime... (Linhart, Le jour où mon père s'est tu)

ou par la locution prépositionnelle quant à :

[54] Ils nous plaisent s'ils sont invisibles. S'ils ne font pas d'histoire. S'ils sont sans histoire. Quant aux autres, ceux qui sont en trop, eh bien, qu'ils cessent d'entrer par la fenêtre alors qu'on les a chassés par la porte. (Storti, L'arrivée de mon père en France) A l'oral spontané, l'élément disloqué peut également survenir en première position, devant $e h$ bien, comme dans cet exemple :

[9a] spk2: en même temps ils faisaient des fêtes extraordinaires sur le palier c' qu'on ferait plus maintenant un très très grand palier et lui eh bien la Russe blanche est partie il a passé ses examens il est entré très jeune à vingt-quatre ans au cabinet de Georges Mandel mais il est resté dans cet immeuble (CFPP2000, 07-03)

Cela permet en [9a] d'introduire après eh bien plusieurs unités syntaxiques prédicatives qui concernent ce thème (la Russe blanche est partie il a passé ses examens il est entré très jeune 
à vingt-quatre ans au cabinet de Georges Mandel mais il est resté dans cet immeuble). Cet exemple :

[14] est ce que (inaud.) pose la question est ce qu'il est pas humain d'accepter le fait qu eles

personnes à qui on a demandé de l'aide eh bien après on leur rende non seulement on l eur rende en leur donnant cette nationalité mais en leur donnant l'occasion de pouvoir $r$ amener leurs proches pour (inaud.) (Corpus : débat sur l'immigration, http://clapi.univ-lyon2.fr)

comprend un thème complexe, pourvu d'une relative (les personnes à qui on a demandé de l'aide), qui se distingue du reste de l'énoncé grâce à la présence d'eh bien.

L'oral spontané ne méconnaît donc pas cette possibilité - élément disloqué puis eh bien mais un autre schéma récurrent se dégage de nos exemples, à la différence de ceux tirés de l'oral représenté : une première prédication existentielle introduite par y avait, on $a, y$, j'avais permet de poser l'existence d'un nouvel objet de discours à propos duquel une deuxième prédication, voire une succession de prédications, initiée par eh bien, donne des caractéristiques (cf. Morel et Danon-Boileau 1998, p. 41-42). Nous avons ainsi une prédication d'existence amorcée par y avait :

[55] spk2 : [...] par exemple dans notre classe de de première $\boldsymbol{y}$ avait une fille qui était fiancée eh bien + non seulement on l'enviait pas mais + on considérait qu'elle était vraiment + que c' était vraiment une catastrophe $+(C F P P 2000,07-05)$

j'ai (cf. aussi l'exemple [45] :

[56] FA17 parce que par rapport je vois aux les miens/ $\boldsymbol{j}$ 'ai deux enfants/

EF oui

FA17 eh bien je vois la différence quand même entre ma fille qui a onze ans et mon fils qui en a quinzel quand même depuis quelque temps elle apprend beaucoup mieux (Corpus : Français des années 80 - entretiens sociolinguistiques, http://clapi.univlyon $2 . f r$ )

L'oral spontané découpe ainsi l'information en deux mouvements: une prédication d'existence et ensuite une ou plusieurs prédications qui apportent des caractéristiques au nouveau référent introduit. Eh bien se trouve à la charnière de ces deux mouvements. Il prend en considération la prédication assertant l'existence du nouvel objet de discours et focalise l'attention sur ce qui en sera dit par la suite. L'exemple [47] :

[47] spk4 :y a quinze eh bien les quinze personnes c'étaient que quinze personnes que du même quartier + et on était deux et j'avais été avec un XX c'était Youssef (CFPP2000, Saint-Ouen-02)

cumule les deux possibilités : la prédication d'existence y a quinze ans et l'élément disloqué les quinze personnes. Peut-être est-ce ainsi parce que la formulation de la prédication d'existence n'est pas aboutie.

Le deuxième cas de figure concerne le cadre spatio-temporel. A l'oral représenté, eh bien peut démarquer du noyau le circonstant, notamment lorsque celui-ci se distingue par sa longueur, comme en [52]. A l'oral spontané nous pouvons trouver comme préfixe un circonstant. Mais il est possible aussi d'évoquer ces circonstances au sein d'une première prédication et d'introduire par eh bien la prédication qui est valide pour ces circonstances. C'est le cas de cet exemple :

[57] ELI_(../.) pas tout à fait un mois: nous c'est les enfants qui passaient pa 'ce avec des cousins/ on louait une maison/ et::: et puis on allait passer euh: pour les vacances pour quand on y va avec les jeunes/

FER les vacances/ $\mathrm{hm}$ 
ELI et puis euh:: MOI un jour j'y suis allée pis j`savais pas qu'j'étais enceinte eh bien j'ai fait:: une fausse couche là bas

SUZ ah

ELI en arrivant (Corpus : Bielefeld, conversations privées, http://clapi.univ-lyon2.fr)

Une série de prédications définit le cadre spatio-temporel qui sera important pour la prédication événementielle que veut apporter le locuteur eh bien j'ai fait:: une fausse couche là bas en arrivant; plus précisément la prédication MOI un jour j'y suis allée fournit le cadre spatio-temporel essentiel pour la compréhension de la suite. Eh bien permet de prendre en considération ce cadre et de délimiter la frontière de la prédication qui est valide pour ce cadre donné précédemment. Dans ce nouvel exemple : Nicel

[58] JAN à ni:ce c'est quand même différent/ une fois nous nous sommes baignés à

\section{KRI hm hm}

JAN eh bien la pla:ge euh:: PUBLIQUE est quand même grande (Corpus : Bielefeld, conversations privées, http://clapi.univ-lyon2.fr)

c'est une fois nous nous sommes baignés à Nice qui fournit le cadre spatio-temporel. Nous voyons que ces prédications comportent des compléments qui ont un rapport avec le temps (un jour, une fois) et le lieu (y, à Nice).

Ainsi, l'oral représenté peut fournir des préfixes complexes, avec une plus grande liberté dans la topologie. A l'oral spontané, plutôt que ne soit modifiée la place d'eh bien dans l'ordre des préfixes, plusieurs prédications apparaissent : les premières permettent de présenter le nouvel objet de discours ou bien les circonstances spatio-temporelles, et les deuxièmes amorcées par eh bien donnent les caractéristiques de ce nouvel objet ou bien inscrivent un événement dans ce cadre spatio-temporel.

\section{Conclusion}

Eh bien, de par son sémantisme lié à l'évaluation du discours, initie des unités syntaxiques prédicatives, ce qui lui permet de se trouver régulièrement dans des configurations discursives adéquates à cette figure d'amorce : il marque une nouvelle formulation, se trouve souvent en début de réplique ou de tour de parole, en début de réponse à une question; il délimite le début du discours rapporté. L'étude de son positionnement topologique en présence d'autres préfixes permet de voir comment l'oral spontané et l'oral représenté utilisent eh bien : dans l'oral représenté, eh bien peut figurer à des emplacements variés dans le groupe des préfixes ; ces derniers peuvent se distinguer par une complexité plus ou moins grande; dans l'oral spontané, deux types de schémas se mettent en place pour permettre à eh bien de continuer à figurer en première position: devant l'unité syntaxique prédicative initiée par eh bien, surviennent d'autres prédications chargées de présenter soit un nouvel objet de discours soit un cadre spatio-temporel : la prédication initiée par eh bien donne des caractéristiques sur ce nouveau référent ou bien est validée dans ce cadre spatio-temporel. Eh bien quant à lui prend en considération l'assertion d'existence ou l'assertion chargée de présenter le cadre spatiotemporel. Ainsi, les marqueurs discursifs basés sur un terme averbal et évaluateurs de discours comme eh bien et bon se trouvent préférentiellement en première position (pour bon, cf. Lefeuvre à paraître b) et ouvrent sur de nouvelles unités syntaxiques.

\section{Bibliographie}

AuChlin A., 1981, « Mais heu, pis bon, ben alors, voilà, quoi! Marqueurs de structuration de la conversation et complétude », Cahiers de linguistique française 2, 141-159. 
Blanche-Benveniste C., 1997, Approches de la langue parlée en français, Gap. Paris : Ophrys (L'Essentiel).

BouchADDAKH S. \& Dostie G., 2007, « Sens lexical, sens grammatical et sens discursif : un continuum? Quelques réflexions à partir du cas de b(i)en », in Larrivée P.(éd.), 2007, Variation et stabilité du français. Des notions aux opérations. Mélanges de linguistique offerts au professeur Jean-Marcel Léard par ses collègues et amis, Paris / Louvain, Peeters, p. 15-32.

Branca S., Fleury S., Lefeuvre F., Pires M., 2009, Constitution et exploitation d'un corpus de français parlé parisien, http://ed268.univ-paris3.fr/syled/ressources/Corpus-ParoleParis-PIII/Presentation.html

BREMOND C., 2002, Les petites marques du discours. Le cas du marqueur métadiscursif bon en français. Thèse de doctorat. Université d'Aix-Marseille I.

Culioli A., 2000 (1990), «Autres commentaires sur bien», Pour une linguistique de l'énonciation, tome 1, Ophrys, Paris, p. 157-168.

Dendale P., 2010, Lexicales (édition 8.0). Bibliographie en ligne d'études linguistiques portant sur des unités lexicales et grammaticales du français, http://webh01.ua.ac.be/lexico/index.html. Copyright (C) 2011 Patrick Dendale

Dostie G. et Pusch C. eds., 2007, « Présentation : les marqueurs discursifs », Les marqueurs discursifs, Langue française, 154, p. 3-12.

FERNANDEZ J., 1994, Les particules énonciatives dans la construction du discours, PUF, Paris

Genette G., 1972, Figures III, Paris, Seuil.

HANSEn M.-B. Mosegaard, 1995, « Marqueurs métadiscursifs en français parlé ; l'exemple de bon et ben », Le Français moderne, 63-1 : p. 20-42.

HANSEN M.-B. Mosegaard, 1998a, « La grammaticalisation de l'interaction ou pour une approche polysémique de l'adverbe bien », Revue de sémantique et pragmatique, 4, p. 111138.

Hansen M.-B. Mosegaard, 1998b, The Function of Discourse Particles. A Study with Special Reference to Spoken Standard French. Amsterdam, John Benjamins. (Chapitre 11 : Eh bien.)

HWANG Y., 1993, "Eh bien, alors, enfin et disons, en français parlé contemporain», L'information

grammaticale, $57: 46-48$.

Le Goffic P., 2006, "Phrase, séquence, période », Modèles Syntaxiques, Bruxelles : Peter Lang, p. 329-356.

LE GofFIC P., 2011, "Phrase et intégration textuelle», Unités syntaxiques et unités prosodiques (Lefeuvre et Moline eds), Langue française, 170.

LEFEUVRE F., 1999, La phrase averbale en français, Paris, L'Harmattan.

LEFEUVRE F., 2007, " Le segment averbal comme unité syntaxique textuelle », in Charolles M., Fournier N., Fuchs C., Lefeuvre F., Parcours de la phrase, Paris, Ophrys, p.143-158 (halshs-00138297).

LEFEUVRE F., 2011, "Bon dans le discours oral: une unité averbale autonome?», in Lefeuvre F. et Behr I., Les énoncés averbaux autonomes entre grammaire et discours, Ophrys, p. 165-185.

Lefeuvre F., Morel M.-A., Teston-Bonnard, 2011, «Valeurs prototypiques de quoi à travers ses usages en français oral ", Neuphilologische Mitteilungen (Bulletin de la Société Néophilologique, Helsinki), p. 37-59

LEFEUVRE Florence, 2013 : «Les réponses aux questions : énoncés fragmentaires, elliptiques ou bien autonomes ? », Pascale Hadermann, Michel Pierrard, Audrey Roig, Dan Van Raemdonck (eds), p. 81-97. 
LEFEUVRE Florence, à paraître : « Bon » à l'oral en tant que préfixe : étude topologique » (Richard ed.).

LEFEUVRE F. et TANGUY N. à paraître : «La représentation de l'oral dans les dialogues dramaturgiques : les énoncés averbaux », in Despierres C. et Krazem M.

Leth ANDERSEN H., 2007, « Marqueurs discursifs propositionnels », in Dostie G. et Pusch C., Les marqueurs discursifs, Langue française, 154, p. 13-28.

LuZZATI D., 1982, « Ben, appui du discours », Le Français moderne, 50-3, p. 193-208.

MAILlarD M., 1974, «Essai de typologie des substituts diaphoriques », Langue française, 21, p. 55-71.

MARTIN R., 1990, "Pour une approche vériconditionnelle de l'adverbe bien », Langue française, 88, p. 80-89.

MichiELS S., 1997, "Etude sémantique et syntaxique du mot bien », Lingvisticae Investigationes, 21, p. 439-441.

Morel M.-A. et Danon-Boileau L., 1998, Grammaire de l'intonation, Paris, Ophrys.

Peroz P., 1992, Systématique des valeurs de bien en français contemporain, Genève, Droz.

Riegel et al., 2009, Grammaire méthodique du français, Paris, PUF.

SIRDAR-ISKANDAR C., "'eh bien!" le russe lui a donné cent francs ", Les mots du discours, chapitre 5 (O. Ducrot et al. eds), p. 160-172.

Teston-Bonnard S., 2006, Propriétés topologiques et distributionnelles des constituants non régis, Application à une description syntaxique des particules discursives (PDI). Thèse de doctorat. Université d'Aix-Marseille I.

VAN BAARDEWYK-RESSEgUIER J., 2002, " Analyse polyphonique de bien, marqueur du discours », Journal of French Language Studies, 12-3, p. 327-349.

VInCENT D., 1993, Les ponctuants de la langue et autres mots du discours, Québec, Nuit Blanche ed.

Winther A., 1985, « Bon, bien, très bien: Ponctuation discursive et ponctuation métadiscursive ", Langue française 65, p. 80-91. 